doi: http://dx.doi.org/10.5892/ruvrv.2012.101.117123

\title{
Etiologia e incidência das fraturas faciais: análise de 152 casos
}

\author{
Oriental Luiz de NORONHA FILHO ${ }^{1}$ \\ Cláudio VALENTE ${ }^{2}$ \\ Kátia KOZLOWSKI ${ }^{3}$
}

\begin{abstract}
${ }^{1}$ Cirurgião-Dentista, Especialista em Saúde da Família, Especialista em Gestão Clínica na Atenção Primária, Especialista em Cirurgia e Traumatologia Bucomaxilofaciais, Especializando em Radiologia e Imaginologia Odontológica-UFJF, Mestre em Saúde Coletiva e Cirurgião-Dentista do PSF Olaria - Cruzília MG.

${ }^{2}$ Professor de anatomia e cirurgia da faculdade de Odontologia do Unifoa, Coordenador do Departamento e Especialização em Cirurgia e Traumatologia Bucomaxillofaciais do Unifoa, Especialista e Mestre em Cirurgia e Traumatologia Bucomaxilofaciais; Doutor em Estomatologia.

${ }^{3}$ Prof ${ }^{a}$. Assistente, Especialista do Departamento de Cirurgia e Traumatologia Bucomaxilofaciais do Centro Universitário de Volta Redonda - RJ.

Autor correspondente: Oriental Luiz de Noronha Filho - Rua Ângela Maria Sciane, 231 - Vila Magalhães - Cruzília MG. E-mail: orientalnfilho@gmail.com

RESUMO: Foi realizado estudo para avaliar a prevalência das fraturas faciais tratadas pela rotina do Curso de Especialização do UniFOA, através do Serviço de Cirurgia e Traumatologia Bucomaxilofacial do Hospital São João Batista, na região sul-fluminense, com sede em Volta Redonda, correlacionando osso fraturado, etiologia, gênero e faixa etária, nos anos de 2009 e 2010. A metodologia aplicada foi por abordagem indutiva com procedimentos estatísticos e descritivos e a técnica a documental indireta com utilização de prontuários dos pacientes atendidos neste período. A amostra totalizou 152 pacientes com fraturas faciais, sendo: 54 com fraturas de mandíbula (35,53\%), 48 com fraturas do complexo zigomático (31,57\%), 46 com fraturas de nariz (30,26\%), dois com fraturas do maxilar (1,32\%) e dois com fraturas específicas do processo alveolar $(1,32 \%)$. As principais etiologias encontradas foram acidentes no trânsito com 54 pacientes $(35,53 \%)$, seguida das quedas acidentais com 50 pacientes $(32,89 \%)$ e a terceira causa mais freqüentes foram agressões físicas em 48 pacientes $(31,58 \%)$. Os indivíduos do gênero masculino se apresentaram em numero de $122(80,26 \%)$ e as do feminino em $30(19,74 \%)$, sendo as faixas mais freqüentes entre 11 a 20 e de 21 a 30 , ambas com 44 casos $(28,94 \%)$ cada e entre 31 e 40 anos com 30 casos (19,73\%). Diante do achado, atentam-se a necessidade de ênfase as medidas educativas e preventivas para a população.
\end{abstract}

Palavras-chave: Face; Ossos Faciais/Lesões; Traumatismos Maxilofaciais; Estudos Retrospectivos.

\section{Etiology and incidence of facial fractures: analysis of 152 cases}

\begin{abstract}
Study was carried through to evaluate the prevalence of the face breakings treated by the routine of the Course of Specialization of the UniFOA, through the Service of Surgery and Bucomaxilofacial Traumatology of the Hospital São João Baptista and Santa Casa de Barra Mansa, in the region south-inhabitant of the state of Rio de Janeiro, with headquarters in Volta Redonda and Barra Mansa, correlating broken bone, etiology, sort and age band, in the years of 2009 and 2010. The applied methodology was for inductive boarding with statistical and descriptive procedures and the documentary technique the indirect one with handbook use of the patients taken care of in this period. The sample totalized 152 patients with face breakings, being: 54 with jaw breaking $(35,53 \%)$, 48 with breaking of the zigomatic complex (31,57\%), 46 with nose breaking (30,26\%), 2 with breaking of maxilar $(1,32 \%)$ and 2 with specific breaking of the alveolar process $(1,32 \%)$. The main joined etiologies had been accidents in the transit with 54 patients $(35,53 \%)$, followed of the accidental falls with 50 patients $(32,89 \%)$ and the third cause more frequent had been physical aggressions in 48 patients $(31,58 \%)$. The individuals of the masculine sort if had presented in number of $122(80,26 \%)$ and of the feminine one in $30(19,74 \%)$, being the bands most frequent between 11 a 20 e de 21 a 30 , to but with 44 cases $(28,94 \%)$ and between 31 and 40 years with 30 cases $(19,73 \%)$. Ahead of the finding, intent it emphasis necessity the educative and preventive measures for the population.
\end{abstract}

Key words: Face; Facial Bones/Injuries; Maxilofacial Injuries; Retrospective Studies. 


\section{INTRODUÇÃO}

É notória a importância de um Serviço de Cirurgia e Traumatologia Bucomaxilofacial em hospitais de grandes municípios, haja vista as fraturas faciais representarem um segmento importante dos problemas de saúde pública, devido a suas seqüelas, as quais podem resultar em sérias inabilidades morfofuncionais. $\mathrm{O}$ Hospital São João Batista em Volta Redonda, e a Santa Casa em Barra Mansa, são as referências sulfluminense no atendimento e tratamento de pacientes vítimas de trauma facial.

A incidência dos traumas bucomaxilofaciais pode variar em virtude da geografia da área onde foi coletada a amostra, da distribuição e das tendências socioeconômicas dentro do universo que contém a amostra, bem como as legislações de tráfego e variações sazonais; características que se acentuam devido ao triângulo geográfico formado pelos estados limites Rio de Janeiro, São Paulo e Minas Gerais.

Não obstante a violência e o trânsito nas grandes cidades, a utilização de motos e bicicletas como meio de locomoção, completam os acidentes em vias publicas, alem das atividades esportivas e as agressões físicas, as quais aumentam a incidência de traumas e fraturas dos ossos da face (DINGMAN; NATVIG, 1983; BARROS, MANGANELLO DE SOUZA, 1993).

$\mathrm{Na}$ região sul do Brasil, Lorenzoni, Moraes (1989) estudaram 124 casos do Hospital de Caridade "Dr Astrogildo de Azevedo" em Santa Maria - Rio Grande do Sul no período entre 1987 e 1989, a mandíbula foi a estrutura óssea mais afetada, com $33.79 \%$ dos casos. Não descreveram etiologia ou gênero.

$\mathrm{Na}$ região sudeste, no Rio de Janeiro, entre 1973 e 1980, Cruz et al., (1982) observaram 1340 pacientes com fraturas faciais do Serviço de Cirurgia Crânio-Maxilo-Facial do Hospital Santa Cruz da Beneficência Portuguesa de Niterói, Rio de Janeiro, em conjunto com a Clinica de Cirurgia Plástica e Reconstrutiva Ivo Pitanguy. Encontraram com maior prevalência as fraturas do zigoma em 498 casos. Não classificaram etiologia, gênero ou faixa etária.

Ainda no estado do Rio de Janeiro, Maia et al., (1987), em pesquisa realizada no Hospital Marcilio Dias na cidade do Rio de Janeiro entre 1985 e 1987, de 178 pacientes com lesões bucomaxilofaciais, a mandíbula foi a estrutura mais afetada com 31 
(55\%) dos casos. Não relataram o gênero.

No estado de São Paulo, Souza et al., (1983) analisaram 450 casos de fraturas faciais do Hospital Matarazzo em São Paulo e encontraram 165 casos de fraturas de nariz. Ainda no estado de São Paulo, Lobo et al., (1998) realizaram um levantamento estatístico de 4792 pacientes atendidos no Serviço de Cirurgia e Traumatologia Bucomaxilofacial do Hospital de Base da Associação Hospitalar de Bauru, em virtude da escassez de trabalhos nacionais e da alta incidência de traumas faciais. No período de 1991 a 1995, encontraram às fraturas do côndilo mandibular como o local e o osso mais fraturado.

$\mathrm{Na}$ região centro-oeste Lobo (1993) analisou 3028 casos de lesões do complexo craniofacial tratados no Hospital da Santa Casa de Misericórdia de Campo Grande - Mato Grosso do Sul entre os anos de 1987 a 1992. Encontrou 726 fraturas do zigoma como a estrutura mais fraturada.

Na região nordeste, Sobreira et al., (2002) avaliaram 306 pacientes do Hospital de Trauma de João Pessoa Paraíba, em 2001, encontraram como mais frequentes, 107 pacientes com fraturas de nariz $(31,47 \%)$.
A prevalência das fraturas faciais em relação às outras do corpo humano é relativamente grande ficando em torno de 9 a 10\%. (PALMA et al., 1995).

\section{MATERIAIS E MÉTODOS}

A metodologia aplicada foi por abordagem indutiva com procedimentos estatísticos e descritivos e a técnica utilizada foi à documental indireta, com utilização de prontuários dos pacientes atendidos nesse período.

Realizou-se levantamento nos arquivos do Serviço de Cirurgia e Traumatologia Bucomaxilofacial do Hospital São João Batista e da Santa Casa de Barra Mansa, dos pacientes atendidos pelo Curso de Especialização em Cirurgia e traumatologia Bucomaxilofacial da UniFOA, no período de 1 de janeiro de 2009 a 31 de dezembro de 2010, observando as fraturas faciais. A pesquisa foi aprovada pelo Comitê de Ética e Pesquisa do HSJB, protocolo $n^{\circ}$ 085/2009.

A amostra foi de 152 pacientes operados pelos alunos do Curso de Especialização do UniFOA, na rotina do Serviço de BMF, após pré-operatório ambulatorial e sob anestesia geral. Os dados foram obtidos das fichas clínicas dos pacientes, as quais ficam em 
arquivos próprios e contem dados sobre o diagnóstico, as causas das fraturas, o gênero e faixa etária do paciente. $\mathrm{O}$ método de abordagem foi indutivo.

\section{RESULTADOS}

Após análise de 152 prontuários, observou-se que as áreas mais afetadas foram: a mandíbula com 54 casos (35,53\%), o complexo zigomático com 48 (31,57\%), o nariz com $46(30,26 \%)$, seguidos do maxilar e do processo alveolar, ambos com apenas dois casos (1,32\%) cada. (Gráfico 1).

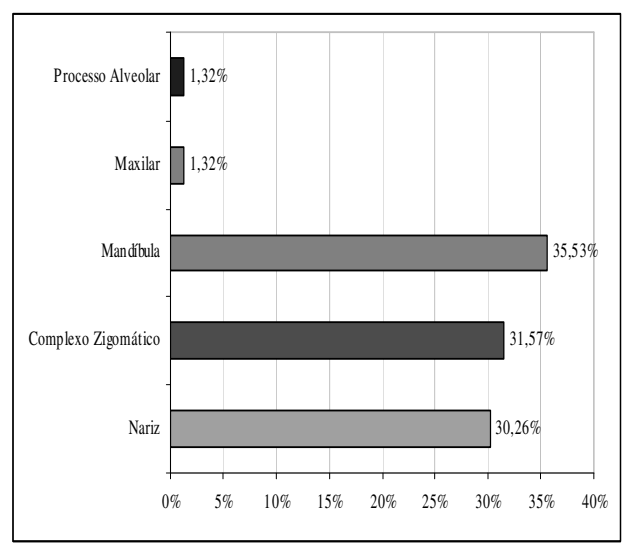

Gráfico 1. Estruturas faciais afetadas

Em relação à etiologia encontrou-se como em conseqüência de: acidentes de trânsito 54 casos $(35,53 \%)$, quedas de alturas 50 casos $(32,89 \%)$ e agressões físicas 48 casos $(31,58 \%)$. (Gráfico 2).

$$
\text { Em relação ao gênero }
$$
encontrou-se 122 casos do gênero masculino $(80,26 \%)$ e 30 feminino (19,74\%). (Gráfico 3).

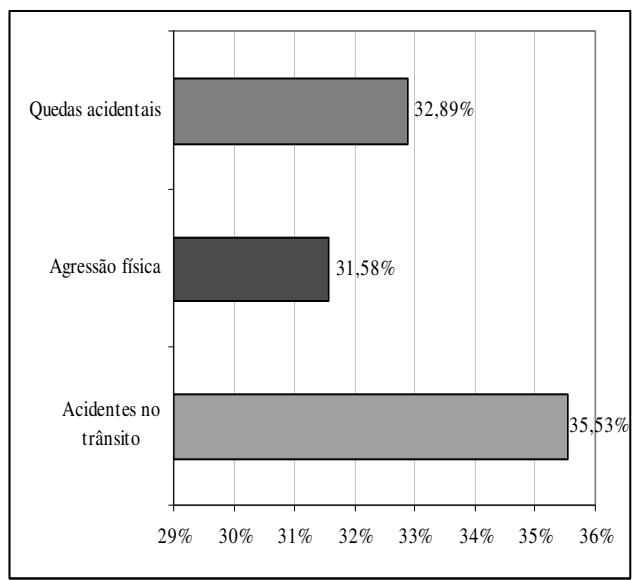

Gráfico 2. Etiologia das fraturas

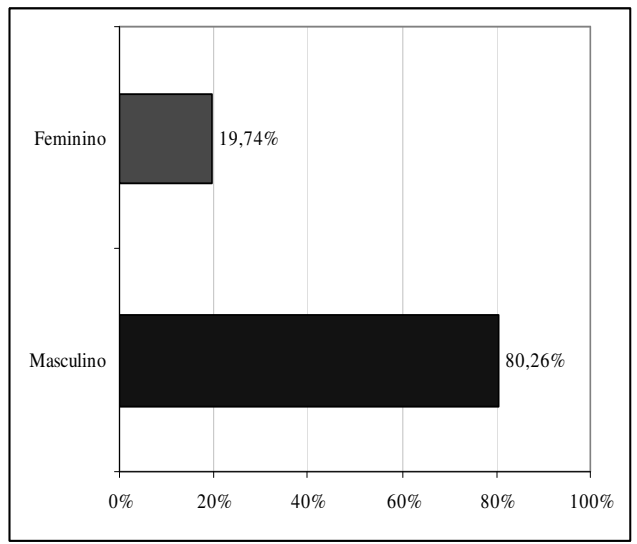

Gráfico 3. Gênero

Em relação à faixa etária houve empate entre as faixas de 11 a 20 e de 21 a 30, ambas com 44 casos $(28,94 \%)$ cada, seguidos da faixa entre 31 a 40 com 30 casos (19,73\%), 41 a 50 anos com 18 casos $(11,84 \%)$, de 51 a 60 e de 61 a 70 anos ambas com 06 casos $(3,94 \%)$ cada, sendo $04 \quad(2,63 \%)$ menores ou iguais a 10 anos, não 
existindo casos acima de 70 anos. (Gráfico 4).

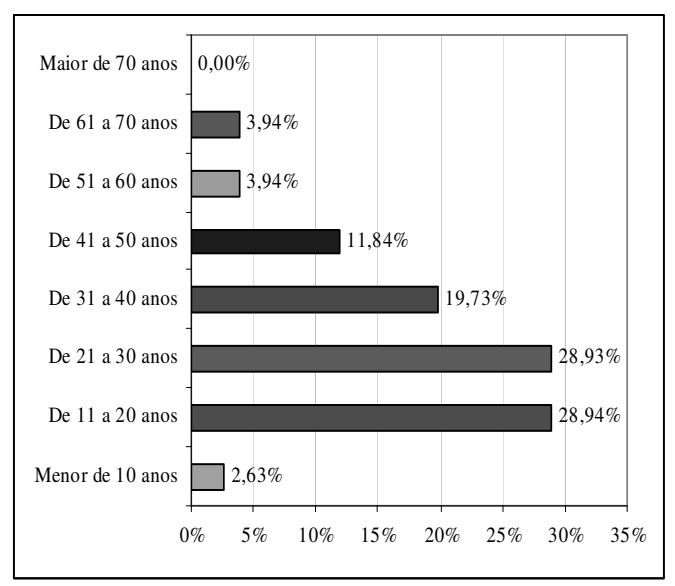

Gráfico 4. Faixa etária

Considerando o tratamento cirúrgico realizado os mais freqüentes foram o cirúrgico cruento com fixação rígida com 98 casos $(64,46 \%)$ e o cirúrgico cruento com tamponamento apresentando 44 casos $(28,94 \%)$ e os menos freqüentes $\mathrm{o}$ incruento com bloqueio intermaxilar e o cruento com fixação semi-rígida, ambas com 4 casos $(2,64 \%)$ cada e o tratamento conservador com apenas um caso (1.32\%). (Gráfico 5).

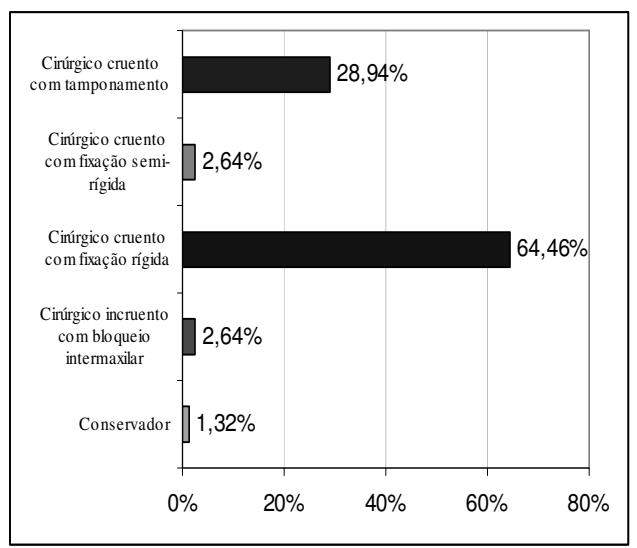

Gráfico 5. Tipo de tratamento realizado

\section{DISCUSSÃO}

Após análise dos 152 pacientes submetidos à cirurgia em busca de tratamento, observou-se que as estruturas faciais mais acometidas foram: a mandíbula com 54 casos (35,52\%), o complexo zigomático com $48(31,57 \%)$ e a fratura nasal com 46 $(30,26 \%)$. Corroborando com os resultados de Lorenzi, Moraes (1989) em relação à ordem de freqüência. Esta ordem discorda de Cruz et al., (1982), os quais encontraram a ordem de complexo zigomático $(41,64 \%)$, seguida da mandíbula $(33,28 \%)$ e do nariz $(28,73 \%)$ e de Souza et al., (1983), o qual encontrou em primeiro o nariz $(36,66 \%)$, seguido do complexo zigomático $(31,33 \%)$ e da mandíbula (23,33\%), corroborando com os resultados de Sobreira et al., (2002). Estes resultados diferentes podem ser justificados pelas diferenças regionais e pela época.

A faixa etária de maior incidência foi entre 11 e 30 anos (57,88\%), havendo empate entre as faixas de 11 a 20 e 21 a 30 anos $(28,94 \%$ cada $)$, tendo a maioria absoluta dos autores pesquisados encontrado maior incidência entre 21 e 30 anos, por ser a faixa etária mais ativa e com menos experiência. 
A etiologia mais frequiente encontrada nesta pesquisa foi a de acidentes automobilísticos, motociclísticos e os ciclísticos, além dos atropelamentos, totalizando $35,52 \%$ dos pacientes, corroborando com os resultados encontrados pelos autores pesquisados. Embora o novo código de trânsito, pela sua atuação mais rigorosa, tenha reduzido o número de pacientes com fraturas faciais, referentes a esta etiologia ainda assim, observam-se dados expressivos a este respeito.

$\mathrm{O}$ gênero mais acometido foi o masculino $(80,26 \%)$, corroborando com todos os autores pesquisados, devido ao fato de estarem mais dispostos as situações de riscos.

\section{CONCLUSÕES}

Com base nos resultados obtidos nesta pesquisa, a mandíbula foi a estrutura mais fraturada com 54 casos $(35,52 \%)$. O principal agente etiológico foram os acidentes automobilísticos, motociclísticos e clisticos e a faixa etária predominante ficou entre 11 e 30 anos, com $57,88 \%$ dos casos. O gênero masculino foi o mais acometido, em uma proporção de 4:1. Medidas legais e educacionais são de grande importância para a prevenção de traumas faciais, devendo ser estabelecidas com todos os rigores; ressaltando a utilização do cinto de segurança, o uso do capacete, respeito aos limites de velocidade, a conscientização sobre o binômio álcool/direção. A Lei Seca veio atuar como medida de prevenção e punitiva para os infratores.

\section{REFERÊNCIAS}

BARROS, J. J.; MANGANELLO DE SOUZA, L. C. Traumatismo Buco Maxilo Facial. São Paulo, Ed. Livraria Rocca Ltda, 1993.

CRUZ, R. L. et al., Abordagem das fraturas de mandíbula em crianças. Sistemática baseada na análise de $\mathbf{4 0}$ casos consecutivos. Rev. Bras. Cir., v.72, n.5, p.228-38, 1982.

DIGMAN, R. O.; NATVIG, P. Cirurgia das fraturas faciais. Trad. Stela M. O. Rossetini, São Paulo, Ed. Santos, 1983, p.43-4.

GANDELMANN, I.A.; CORTEZZI,W. - Incidência e tratamento das lesões traumáticas da mandíbula, maxilofacial e as estruturas dentárias na cidade do Rio de Janeiro, de 1976 a 1982. Rev. Gauc. Odont 43 (1) 32-39, jan., 1986.

\section{LOBO, A. R. - Incidência das} fraturas faciais na região de Mato Grosso do Sul, [Dissertação de Mestrado]. Pelotas: Faculdade de Odontologia de Pelotas - RS; 1993.

LOBO, S. E.; MARZOLA, C.;

TOLEDO-FILHO, J. L. et.al. Incidência e tratamento de fraturas do côndilo da mandíbula no Serviço de Cirurgia e Traumatologia 
Bucomaxilofacial da Faculdade de Odontologia de Bauru da Universidade de São Paulo e Associação Hospitalar de Bauru, no período de 1991 a 1995. Rev. Odonto Ciênc., v.13, n.25, p.7-38, jun., 1998.

LORENZONI, J.A.; MORAES,C.J. Traumatismos faciais; revisão de 124 casos. Rev. Bras Cien Saúde; v.15, n. 12, p.97-105, 1989.

MAIA, J.S.; GARRITANO, C.A.; DAMASCENO L.M.G. - Conduta nos traumatismos bucomaxilofaciais por arma de fogo. Rev Naval Odont v.1, n.2, p.20-22, 1987.

PALMA, V. C. et al. Frequência de fraturas faciais em pacientes atendidos num serviço hospitalar. Rev. Odont. Univ. São Paulo, v.9, n.2, p.121-6, 1995.

REIS, L. F.; MARZOLA, C.; TOLEDO-FILHO, J. L. et al. Prevalência das fraturas faciais, na região de Bauru, no período de janeiro de 1991 a dezembro de 1995. Rev. Odonto Cienc., v.16, n.34, p.23140, set-dez., 2001.

SANTOS, J. P.; MARZOLA, C.; TOLEDO-FILHO, J. L. Incidência e tratamento de fraturas do côndilo da mandíbula no Serviço de Cirurgia e Traumatologia Buco Maxilo Facial de Bauru do Hospital de Base de Bauru no período de 1996-1998. Monografia apresentada como parte dos requisitos exigidos para conclusão da Residência em Cirurgia e Traumatologia Buco Maxilo Facial. Faculdade de odontologia de Bauru da USP. Bauru, 1998.

SANTOS JÚNIOR, P. V. Incidência de fraturas faciais no município de São José dos Campos, SP. Rev. Odontol. UNESP, SP, v.21, n.1, p.215-21, 1992.
SOBREIRA, T. ET AL. Prevalência de Traumatismos Bucomaxilofaciais em João Pessoa - Paraíba - Brasil. Rev. Bras. Ciências da Saúde, v. 6, n.1, p.2532, abr., 2002.

SOUZA, L. C. M.; FISCHMAN, R.; SILVEIRA, M. E. et al. Estudo de 450 casos de fraturas de ossos da face. Rev. Assoc. Paul. Cirur. Dent., v.37, n.3, p.256-60, maio-jun., 1983.

VALENTE, C. Emergências em bucomaxilofacial. 1. ed., Rio de Janeiro, Ed. Revinter, 1999. 